\title{
Einfluss von Rotem Ginseng auf ein Krafttraining bei älteren Erwachsenen: Eine randomisierte placebokontrollierte Doppelblindstudie
}

\author{
Nadja Schott ${ }^{1}$, Sabine Konietzny², Christoph Raschka² \\ 1 Institut für Sportwissenschaft, Justus Liebig Universität, DE-Giessen \\ 2 Institut für Sportwissenschaft, Johann Wolfgang Goethe-Universität, DE-Frankfurt
}

m Jahr 2050 wird in Deutschland die Zahl der über 65-Jährigen auf mehr als 25 Millionen anwachsen (36\% der Gesamtbevölkerung). Die durchschnittliche Lebenserwartung wird auf 82 Jahre bei den Männern und 87 Jahre bei den Frauen ansteigen [1]. Jedoch kann nicht davon ausgegangen werden, dass mit ansteigender Lebenserwartung die Lebensqualität in gleichem Masse zunimmt. Vielmehr bedeutet momentan ein längeres Leben eher ein längeres Leben mit chronischen Krankheiten [2]. Die Bestimmung des notwendigen Umfangs und der Wirkmechanismen von Sport und Bewegung als Einflussgrössen, aber auch die unterstützende Wirkung von Nahrungsergänzungsmitteln auf Gesundheit, Funktionalität, Lebensqualität und Unabhängigkeit in dieser Altersgruppe gewinnt an Bedeutung, je mehr Menschen immer älter werden [3].

\section{Krafttraining im Alter}

Krafttraining besitzt aus sportmedizinischer und trainingswissenschaftlicher Sicht eine hohe Wirksamkeit als Ressource auf eine adäquate Lebensbewältigung auch im Alter [4]. Altersbedingte Verluste in der Muskelkraft gehen häufig einher mit einer eingeschränkten funktionalen Mobilität wie z.B. das Aufstehen von einem Stuhl oder einer erhöhten Sturzgefahr [5]. Da insbesondere die Aktivitäten des täglichen Lebens für Senioren immer schwieriger $\mathrm{zu}$ bewältigen sind, führt dies zu einer Einschränkung des Lebensstiles und damit wiederum zu einer Einschränkung der gesamten körperlichen Aktivität. Ein Krafttraining
Hintergrund: Regelmässige Bewegung bietet eine der besten Möglichkeiten, die Anzahl an Jahren unabhängigen und selbständigen Lebens bei älteren Menschen zu verlängern. Pflanzliche Mittel werden seit langem genutzt, um die Leistungsfähigkeit zu steigern, jedoch liegen kontrollierte Studien bisher nur für jüngere Menschen vor. Studienziel: Ziel der Studie ist es, den Einfluss einer täglichen Gabe von 1'200 mg Rotem Ginseng (Ginsenosidgehalt 8\%) auf die Effekte eines 60-minütigen, wöchentlich durchgeführten Krafttrainings bei älteren Erwachsenen zu untersuchen. Studiendesign: Randomisierte und placebokontrollierte Doppelblind-Studie. Methoden: 24 Männer und 46 Frauen (Alter = 64.7 Jahre \pm 5.5 ; Körpergrösse $=168 \mathrm{~cm} \pm 7.6$; Körpergewicht $=71.1 \mathrm{~kg} \pm 13.1$; Körperfettprozentsatz $=28.5 \% \pm 5.5$; sportliche Aktivität $=321$ Minuten \pm 181 pro Woche) wurden randomisiert einer der vier Untersuchungsgruppen (Ginseng, Krafttraining, Ginseng \& Krafttraining, Placebo) zugeteilt. Die Studiendauer betrug 12 Wochen bei einer Medikation Roter Ginseng von 1'200 mg (= 2 × 2 Kapseln) pro Tag. Pre und post wurden klinische Parameter, die Befindlichkeit sowie Kraftparameter der grossen Muskelgruppen erhoben. Die Datenanalyse wurde mit Varianzanalysen mit Messwiederholungen durchgeführt. Ergebnisse: Unter der Medikation mit Rotem Ginseng konnten für die klinischen Parameter Pulsfrequenz und Körperfettprozentsatz positive Effekte festgestellt werden. Ebenso ergaben sich Leistungsverbesserungen für die Kraftparameter. Mehr als 80\% der Studienteilnehmer mit Ginsengeinnahme berichteten von einer Vitalitätszunahme. Schlussfolgerungen: Die Ergebnisse der vorliegenden Studie stützen die deskriptiven positiven Effekte von Rotem Ginseng auf klinische, Befindlichkeits- und Kraftparameter im Verlauf eines 12-wöchigen Krafttrainings bei älteren Erwachsenen.

Schlüsselwörter: Roter Ginseng, Krafttraining, Körperfett, Senioren

\section{Red Ginseng Enhances the Effectiveness of Strength Training in Elderly: A Randomized Placebo-Controlled Double-Blind Trial}

Background: Regular physical activity offers one of the greatest opportunities to extend years of active independent life for older persons. Herbs have been used throughout history to enhance physical performance, but scientific scrutiny with controlled clinical trials has only been used to study such effects in younger adults. Aim of the study: To examine the effects of a daily dose of $1200 \mathrm{mg}$ red ginseng supplementation (ginsenoside $8 \%$ ) alongside a weekly strength training session of 60 minutes in elderly. Design: Randomized, placebo-controlled, double-blinded study. Methods: 24 men and 46 women (age $=$ 64.7 years \pm 5.5 ; height $=168 \mathrm{~cm} \pm 7.6$; weight $=71.1 \mathrm{~kg} \pm 13.1$; body fat percentage $=$ $28.5 \% \pm 5.5$; physical activity $=321$ minutes \pm 181 per week) were randomly allocated to 4 groups (ginseng, strength training, ginseng \& strength training, placebo). The participants on supplements, received either $1200 \mathrm{mg}$ red ginseng or placebo daily for 12 weeks ( $2 \times 2$ capsules). The main outcome measures were clinical parameters, self-assessed well-being, as well as strength of the large muscle groups; these were measured at baseline and at 12 weeks. Data analysis used repeated measures of variance. Results: Statistically significant reductions in heart rate and body fat percentage were observed in the "ginseng"-groups after 12 weeks of treatment; strength values were higher. More than $80 \%$ of the subjects under ginseng medication reported an increase in vitality. Conclusions: These data suggest that red ginseng can support improvements in clinical parameters, well-being and muscle strength after a 12 week strength training program in elderly.

Key words: Red ginseng, strength training, body composition, elderly 
zeigt seine Wirkung nicht nur in der Verbesserung physiologischer Komponenten wie die Vergrösserung von Muskelgrösse und -kraft, der Reduktion des Blutdrucks, der Verbesserung der Blutzusammensetzung, der Abschwächung von Rückenschmerzen, der Stärkung der Knochendichte, der Erleichterung von arthritischen Beschwerden, der Minderung von Depressionen, der Verbesserung postkoronarer Leistung sowie der Verminderung der Sturzgefahr, sondern vor allem in seiner Moderatorwirkung auf Lebensstil und Lebensqualität [3, 6]. Bei der subjektiven Einschätzung der Verschlechterungen motorischer Fähigkeiten wird die Kraft am häufigsten genannt. Während und nach dem Training sprechen Teilnehmer von Krafttrainingskursen vielfach von langen Regenerationsphasen bzw. Müdigkeit. Dies führt in vielen Fällen zu einem Abbruch des Trainings [7].

\section{Ginseng (Panax ginseng)}

Zusammen mit der sibirischen Taigawurzel (Eleutherococcus senticosus), dem südamerikanischen Mate (Ilex paraguariensis) und dem afrikanischen Kolabaum (Cola nitida) gehört der aus Ostasien, Nepal, Mandschurei, Korea, Ostindien, Japan, Kanada, Süd-USA stammende Panax ginseng zur phytotherapeutischen Gruppe der Adaptogene [8]. Diese Substanzen steigern die Fähigkeit zur Abwehr von Stressoren und optimieren die Anpassung an aussergewöhnliche Belastungen, indem sie die Widerstandskraft gegen Noxen physikalischer, chemischer und biologischer Art unspezifisch erhöhen. Dies erfolgt unabhängig von der Art des pathologischen Zustands, fokussiert aber vor allem auf geringere Störungen der Körperfunktionen (Antistress-Wirkung gegenüber Stressoren nichtinfektiöser Art, Immunstimulation). Zusätzlich kommen nootrope (=Verbesserung der höheren integrativen Hirnfunktionen) und anabole Effekte (Aktivierung des Aufbaustoffwechsels) zum Tragen [8]. Die Wirkung der Panax ginseng-Extrakte wird von der Mehrheit der Pharmakologen auf die Ginsenoside zurückgeführt, Triterpensaponine, von denen mindestens 10 verschiedene Einzelverbindun- gen existieren. Der Gehalt an Ginsenosiden hängt von Anbaugebiet, Pflanzenalter und den jeweiligen Wurzelteilen ab und beträgt in der getrockneten Droge 2-3\%, manchmal bis $6 \%$. Die Ginsengwurzel stammt von 4- bis 6-jährigen Pflanzen und kommt als „Weisser Ginseng“ oder als „Roter Ginseng“ in den Handel. Beim Weissen Ginseng wird nach der Ernte die Korkschicht der Wurzel entfernt ("geschält") und dann die Wurzel getrocknet, den Roten Ginseng erhält man durch Hitzebehandlung der frisch gegrabenen Wurzeln (Dampfsterilisation über 2-3 Std. bei $120^{\circ}-130^{\circ} \mathrm{C}$ ) zwecks Abtötung von anhaftenden Mikroorganismen [9]. Indikationen sind Erschöpfungszustände, Rekonvaleszenz, Stärkung und Kräftigung bei Müdigkeit und Schwäche, nachlassende Leistungsund Konzentrationsfähigkeit [8]. Als Tonikum wird Ginseng vor allem aus traditionellen Gründen in verschiedenen orientalischen Ländern, China, Japan und Korea eingesetzt. Deswegen wird im ganzen Orient Ginseng auch auf dem Sportsektor gerne von Athleten zur Verbesserung von Kraft, Kraftausdauer und Regeneration eingesetzt.

\section{Einfluss von Ginseng auf die motorische Leistungsfähigkeit beim Menschen}

Untersuchungen an Menschen sind spärlich und zeigen unterschiedliche Resultate [10]. So ergab sich nach ENGELS und WIRTH [11] nach einer 8wöchigen Therapie bei Gesunden keine signifikante Verbesserung der Ausdauerleistungsfähigkeit, und es konnten keine ergogenen Effekte bei der Verbesserung von submaximaler bzw. maximaler Ausdauer durch Panax ginseng C.A. Meyer gezeigt werden. PIERALISI und Kollegen [12] untersuchten in einer Doppelblindstudie 50 gesunde Sportlehrer, die über einen Zeitraum von sechs Wochen zwei Kapseln Ginseng oder zwei Kapseln Placebo erhielten. Ihre Ergebnisse wiesen bei der Ginsenggruppe hingegen eine höhere Arbeitsleistung auf dem Laufband auf, zusammen mit einer erhöhten $\mathrm{O}_{2}$ max. Die $\mathrm{O}_{2}$-Aufnahme, die Plasmalaktatwerte, die Ventilation, der $\mathrm{CO}_{2}$ Verbrauch und die Herzfrequenz wa- ren signifikant niedriger nach der Ginseng-Einnahme.

Nur wenige Studien existieren zum Einfluss von Ginseng auf die Muskelkraft. McNaughton et al. [13] verteilten 15 Frauen und 15 Männer auf eine Placebogruppe, eine Chinesische-Ginseng-Gruppe (1g/Tag) sowie eine Russische-Ginseng-Gruppe (1g/Tag). Nach einer 6-wöchigen Periode wurde die Medikation für die beiden Gruppen getauscht. Die Autoren konnten signifikante Verbesserungen in der $\mathrm{Vo}_{\max }$ sowie in der Erholungsrate der Herzfrequenz für die Gruppe mit dem Chinesischen Ginseng feststellen. Zwar konnten keine Verbesserungen in der Handkraft, jedoch Zunahmen im M. Pectoralis sowie M. quadriceps bestätigt werden. KANG und Kollegen [14] überprüften die Auswirkung von $20 \mathrm{~g}$ Ginsengwurzelextrakt auf die hormonelle Antwort auf ein Krafttrainingsprogramm mit jeweils $3 \times 7$ Wiederholungen maximal an 8 Übungsstationen (Bankdrücken, Beinpresse u.s.w.) und fanden bei 8 Studenten in dieser placebokontrollierten randomisierten Studie keine signifikanten Unterschiede bezüglich STH, Testosteron, Kortisol, IGF-1 in den zwei Stunden nach Belastungsende.

Die tatsächliche Wirkung von Ginseng-Präparaten kann trotz einer Vielzahl an Studien nicht widerlegt, aber auch nicht eindeutig bestätigt werden. BAHRKE und MORGAN [15] kommen zu dem Schluss, dass methodologische Überlegungen deutlicher in die Planung miteinbezogen werden müssen. So kritisieren sie die ungenauen Angaben bzgl. der Ginsengprodukte, die unterschiedliche Dosierung sowie Reinheit der Ginseng-Präparate, die Einnahme der Präparate, die Variation der Testzeiträume und die häufig geringe Teilnehmerzahl. Nicht alle Studien sind placebo-kontrollierte Doppelblindstudien. Statistische Kriterien wie Validität, Reliabilität und Objektivität müssen gewährleistet sein. Die Testpersonen müssten genauer überprüft werden bzgl. ihrer Lebens-, Essgewohnheiten und ihrem Sportverhalten.

Ziel dieser Studie war es deshalb, den Einfluss der Gabe eines definierten Ginseng-Präparates (Roter Ginseng) auf die Effekte eines 60-minütigen, wö- 
chentlich durchgeführten Krafttrainings im Rahmen einer randomisierten Doppelblindstudie mit 70 Erwachsenen im Alter von 52 bis 82 Jahren zu untersuchen. In der hier vorliegenden Studie sollten nun einige der bisher nur wenig beachteten Kriterien umgesetzt worden. Die Probandenzahl beläuft sich auf 70 Testpersonen. Die Studiendauer betrug 12 Wochen. Die Testpersonen erhielten über diesen Zeitraum 1200mg Roten Ginseng ( 4 Kapseln $=96 \mathrm{mg}$ Ginsenoside, Ginsenosidgehalt $8 \%$ ) pro Tag verabreicht. Es sollten dadurch die Effekte der angegebenen Ginsenggabe auf die Kraftfähigkeiten der grossen Muskelgruppen bei 70 Senioren im Alter von 52 bis 88 Jahren zu untersuchen.

\section{Material und Methoden}

\section{Untersuchungsstichprobe}

An der Untersuchung nahmen insgesamt 70 Personen (24 Männer und 46 Frauen) teil (Tab. 1). Alle Probanden waren gesund und nahmen keine Medikamente ein, die das Untersuchungsergebnis verfälschen könnten. Die Versuchsgruppen unterschieden sich nicht signifikant hinsichtlich des Alters, der Grösse und des Gewichts, des Körperfetts sowie des Grades der sportlichen Aktivität auf dem 5\%-Niveau.

\section{Design und Ablauf}

Eingangsmessung: Die Probanden wurden randomisiert den Gruppen Placebo, Ginseng, Krafttraining sowie Ginseng \& Krafttraining zugeteilt. Zur Absicherung der internen Validität wurden nicht nur eine, sondern drei Experimentalgruppen sowie eine zusätzliche Kontrollgruppe ausgewählt. Zunächst waren alle Gruppen mit je 20 Probanden besetzt. Aufgrund gesundheitlicher Probleme im Laufe des Trainings sowie zu geringer Trainingshäufigkeit mussten 12 Versuchspersonen aus den Analysen ausgeschlossen werden. Eine Verzerrung durch diese Dropouts konnte nicht festgestellt werden. Nach einer ausführlichen Untersuchung durch einen Arzt konnten keine medizinischen Bedenken gegen eine Ginsengeinnahme sowie die Teilnahme an einem Krafttraining (u.a. keine gesund-

Tab. 1. Stichprobencharakteristika

\begin{tabular}{|lrrrr|}
\hline & $\begin{array}{r}\text { Placebo } \\
(\mathrm{n}=21) \\
\chi \pm \mathrm{s}\end{array}$ & $\begin{array}{r}\text { Ginseng } \\
(\mathrm{n}=19) \\
\chi \pm \mathrm{s}\end{array}$ & $\begin{array}{r}\text { Krafttraining } \\
(\mathrm{n}=10) \\
\chi \pm \mathrm{s}\end{array}$ & $\begin{array}{r}\text { Krafttraining \& } \\
\text { Ginseng }(\mathrm{n}=20) \\
\chi \pm \mathrm{s}\end{array}$ \\
\hline Alter & $64.8 \pm 5.4$ & $65.6 \pm 5.9$ & $65.3 \pm 4.4$ & $63.4 \pm 6.0$ \\
Grösse (cm) & $166.9 \pm 7.5$ & $165.9 \pm 7.0$ & $168.3 \pm 4.9$ & $171.5 \pm 8.5$ \\
Gewicht (kg) & $70.7 \pm 12.3$ & $70.1 \pm 15.7$ & $68.1 \pm 10.7$ & $74.0 \pm 12.8$ \\
Körperfett (\%) & $28.7 \pm 4.2$ & $28.3 \pm 4.9$ & $28.0 \pm 8.0$ & $28.8 \pm 6.2$ \\
Sportliche Aktivität & & & $369.0 \pm 116.8$ & $288.8 \pm 177.8$ \\
(min/Woche) & $279.5 \pm 141.0$ & $378.2 \pm 234.8$ & & \\
\hline
\end{tabular}

heitlichen Einschränkungen, keine störende Medikamenteneinnahme) festgestellt werden. Es wurden verschiedene klinische Parameter (u.a. Herzfrequenz, Blutdruck, Grösse, Gewicht, Körperkomposition) ermittelt. Die Körperkomposition wurde über eine Hautfaltendickenmessung an 10 Stellen erhoben. Die Probanden wurden über die Ziele der Studie informiert, die Einverständniserklärung wurde unterschrieben. Im Pretest wurden nachfolgend genannte Tests an standardisierten Kraftgeräten durchgeführt.

Brustpresse - grosser Brustmuskel, Vorderer Teil des Deltamuskels, Langer Kopf des Triceps, Medialer Kopf des Triceps

Rudergerät - hinterer Teil des Deltamuskels, Untergrätenmuskel, kleiner runder Armmuskel, Kapuzenmuskel, Rautenmuskel

Latziehen hinter dem Rücken - breiter Rückenmuskel, grosser runder Armmuskel, Biceps, Armbeuger, Oberarmspeichenmuskel, Rautenmuskel, untere Fasern des Kapuzenmuskels

Beinpresse - vierköpfiger Schenkelmuskel, Gesässmuskel, Kniebeuger, Adduktoren

Kniestrecker - vierköpfiger Schenkelmuskel

Bauchpresse - äusserer schräger Bauchmuskel, gerader Bauchmuskel, Spanner der Oberschenkelbinde

Da häufig die unbekannte Testsituation $\mathrm{zu}$ den Verbesserungen in den Kraftwerten führt [16] sowie aufgrund des Alters und der Belastungen bei Krafttests, wurde nicht das 1-Wiederholungsmaximum ermittelt. Vielmehr sollten die Probanden eine Einschätzung auf der BorG-Skala [17] abgeben, wann fünf Wiederholungen als sehr anstrengend empfunden werden.

Ginsenggabe: In dieser Studie wurde das Handelspräparat „Roter Ginseng Gintec ${ }^{\circledR}$ “ der Firma Gintec/Königstein i. Taunus verwendet. Die Testpersonen erhielten über den Zeitraum von 12 Wochen pro Tag 1200mg Roten Ginseng ( 4 Kapseln $=96 \mathrm{mg}$ Ginsenoside, Ginsenosidgehalt 8\%). Das Placebo wurde in Aussehen und Inhalt ohne den Ginsengwirkstoff produziert. Die Probanden sollten je zwei Kapseln morgens und abends vor dem Frühstück bzw. Abendessen einnehmen. Die Ernährungsgewohnheiten sollten während der Testphase nicht umgestellt werden.

In den Experimentalgruppen III (Krafttraining + Ginseng) und II (Krafttraining) erfolgte die Gabe des Präparates randomisiert (blind), so dass die Teilnehmer in EG III das Ginseng-Präparat erhielten und in EG II ein Placebo. Die Einteilung in EG III und II war den Teilnehmern nicht bekannt. In EG I sowie der KG erfolgte die Gabe des Präparates ebenfalls randomisiert (blind).

Treatment: Einmal pro Woche absolvierten die Probanden der Experimentalgruppen II und III ein 60-minütiges erschöpfendes Trainingsprogramm. Dabei wurden die Muskelgruppen Arme, Schultern, Brustmuskeln, Rücken, Beine, Bauch in das Training miteinbezogen. Es wurden insgesamt 8 bis 10 Übungen in 3 Sätzen mit je 10 bis 15 Wiederholungen mit mittleren Intensitäten (Gewichten) durchgeführt.

Ausgangsmessung: 12 Wochen nach dem Eingangstest mussten die Probanden erneut einen Fragebogen zur 
Tab. 2. Kovarianzanalyse (Alter, Geschlecht) mit Messwiederholungen und dem Faktor Untersuchungsgruppe. $T=p<0.10 ;{ }^{*}=p<0.05$; ${ }^{*}=p<0.01$

\begin{tabular}{|c|c|c|c|c|c|}
\hline & \multirow[b]{2}{*}{$\mathbf{n}$} & \multirow{2}{*}{$\begin{array}{l}\text { MZP1 } \\
\chi \pm s\end{array}$} & \multirow{2}{*}{$\begin{array}{l}\text { MZP2 } \\
\chi \pm s\end{array}$} & \multicolumn{2}{|c|}{ Statistische Analysen } \\
\hline & & & & $F, p$ & $\mathrm{Eta}^{2}$ \\
\hline \multicolumn{6}{|l|}{ Puls } \\
\hline Placebo & 21 & $80.71 \pm 15.82$ & $76.14 \pm 13.05$ & $\mathrm{~F}_{\text {Puls }}=12.14 * *$ & .159 \\
\hline Ginseng & 19 & $85.95 \pm 13.53$ & $78.00 \pm 13.06$ & $\mathrm{~F}_{\text {Puls } \times \text { Sex }}=7.12^{* *}$ & .100 \\
\hline Krafttraining & 10 & $79.20 \pm 12.92$ & $76.40 \pm 11.90$ & $\mathrm{~F}_{\text {Puls } \times \text { Alter }}=12.05^{* *}$ & .158 \\
\hline Ginseng \& Krafttraining & 20 & $90.00 \pm 12.27$ & $79.40 \pm 11.25$ & $\mathrm{~F}_{\text {Puls }} \times$ Gruppe $=2.28^{\top}$ & .097 \\
\hline \multicolumn{6}{|l|}{ Systolischer Blutdruck } \\
\hline Placebo & 21 & $148.24 \pm 18.83$ & $143.33 \pm 14.23$ & $\mathrm{~F}_{\mathrm{SBD}}=1.12$ & .017 \\
\hline Ginseng & 19 & $146.74 \pm 19.87$ & $141.05 \pm 16.70$ & $\mathrm{~F}_{\mathrm{SBD} \times \mathrm{Sex}}=5.09 *$ & .074 \\
\hline Krafttraining & 10 & $141.20 \pm 26.14$ & $136.30 \pm 19.20$ & $\mathrm{~F}_{\mathrm{SBD} \times \text { Alter }}=0.16$ & .003 \\
\hline Ginseng \& Krafttraining & 20 & $136.75 \pm 15.36$ & $138.70 \pm 12.23$ & $\mathrm{~F}_{\mathrm{SBD} \times \mathrm{Gruppe}}=0.79$ & .036 \\
\hline \multicolumn{6}{|l|}{ Diastolischer Blutdruck } \\
\hline Placebo & 21 & $92.14 \pm 12.24$ & $89.14 \pm 9.19$ & $F_{D B D}=1.49$ & .023 \\
\hline Ginseng & 19 & $85.95 \pm 7.26$ & $88.84 \pm 10.16$ & $\mathrm{~F}_{\mathrm{DBD} \times \mathrm{Sex}}=0.01$ & .000 \\
\hline Krafttraining & 10 & $89.20 \pm 16.10$ & $86.90 \pm 13.84$ & $\mathrm{~F}_{\mathrm{DBD} \times \text { Alter }}=1.95$ & .030 \\
\hline Ginseng \& Krafttraining & 20 & $89.50 \pm 15.07$ & $91.35 \pm 11.39$ & $\mathrm{~F}_{\mathrm{DBD}} \times$ Gruppe $=0.95$ & .042 \\
\hline \multicolumn{6}{|l|}{ Körperfett } \\
\hline Placebo & 21 & $28.74 \pm 4.18$ & $29.35 \pm 4.41$ & $\mathrm{~F}_{\mathrm{Fett}}=0.32$ & .005 \\
\hline Ginseng & 19 & $28.33 \pm 4.92$ & $28.82 \pm 5.22$ & $\mathrm{~F}_{\mathrm{Fett} \times \mathrm{Sex}}=0.02$ & .000 \\
\hline Krafttraining & 10 & $28.03 \pm 7.98$ & $28.57 \pm 8.23$ & $\mathrm{~F}_{\mathrm{Fett}} \times$ Alter $=0.29$ & .005 \\
\hline Ginseng \& Krafttraining & 20 & $28.78 \pm 6.17$ & $27.67 \pm 6.94$ & $\mathrm{~F}_{\text {Fett } \times \text { Gruppe }}=3.72^{*}$ & .149 \\
\hline
\end{tabular}

Befindlichkeit ausfüllen. Darüber hinaus wurden die Tests aus der Eingangsmessung (klinische Parameter, Krafttests) wiederholt.

\section{Datenanalyse}

Mittelwerte und Standardabweichungen wurden für alle demographischen und anthropometrischen Angaben sowie für die Kraftparameter berechnet. Die Unterschiede zwischen und in den Gruppen wurden mit Varianzanalysen mit Messwiederholungen für alle Krafttests durchgeführt und zwar mit der unabhängigen Variablen 'Gruppenzugehörigkeit' mit den Kovariaten 'Geschlecht' und 'Alter'. Das $\alpha$-Level wurde für alle statistischen Analysen auf 0.05 festgesetzt. Darüber hinaus wurden die prozentualen Veränderungen zur anschaulicheren Darstellung berechnet.

\section{Ergebnisse}

\section{Klinische Parameter}

In Tabelle 2 werden die Ergebnisse der Varianzanalyse mit dem Messwiederholungsfaktor Zeit, der unabhängigen
Variablen 'Untersuchungsgruppe' sowie den Kovariaten 'Alter' und 'Geschlecht' für die klinischen Parameter dargestellt. Für die Pulsfrequenz lassen sich neben der signifikanten Absenkung des Ruhepulses von Messzeitpunkt 1 zu 2 eine Tendenz in Bezug auf die Untersuchungsgruppen feststellen. Die deutlichste Absenkung zeigten die Gruppe Ginseng mit 11.8\% sowie die Gruppe Ginseng \& Krafttraining mit $14.8 \%$. Keine signifikanten Effekte lagen für die Zwischensubjekteffekte Untersuchungsgruppe, Alter und Geschlecht vor. Für den Blutdruck ergeben sich nur deskriptive Schwankungen. Ein signifikanter Einfluss der Untersuchungsvariablen lässt sich nicht feststellen. Einzig die Gruppe Ginseng \& Krafttraining hat ihren Körperfettprozentsatz um 5\% absenken können, nur marginale Veränderungen gab es für die anderen Untersuchungsgruppen.

\section{Veränderungen durch Ginseng}

Nach Abschluss der Trainingsphase wurden die Teilnehmer nach den subjektiv empfundenen Veränderungen befragt (Mehrfachnennungen waren möglich). Während keiner der Probanden in der Krafttrainingsgruppe und nur 2 Teilnehmer in der Placebogruppe überhaupt von Veränderungen durch Ginseng sprechen, sind dies 17 Personen in der Ginseng- und 15 Personen in der Gruppe Ginseng \& Krafttraining. In Abbildung 1 werden deshalb nur die von den Probanden der Gruppe Ginseng sowie der Gruppe Ginseng \& Krafttraining geschilderten Veränderungen durch das Treatment dargestellt. Probanden, die Ginseng einnahmen, berichteten von Vitalitätszunahme $(\mathrm{n}=16)$, Gewichtszunahme $(\mathrm{n}=2)$, Verdauungsproblemen $(n=1)$ und einer Verbesserung der Hautelastizität $(n=2)$. Die zentrale Veränderung in der Gruppe Ginseng \& Krafttraining war ebenfalls die Vitalität $(n=12)$. Weiterhin berichteten sechs Teilnehmer dieser Gruppe eine Gewichtszunahme, welche sicherlich auch auf das Krafttraining zurück zu führen ist.

\section{Kraftparameter}

Univariate Varianzanalysen ergaben keine signifikanten Unterschiede in den Kraftparametern zu Beginn der 


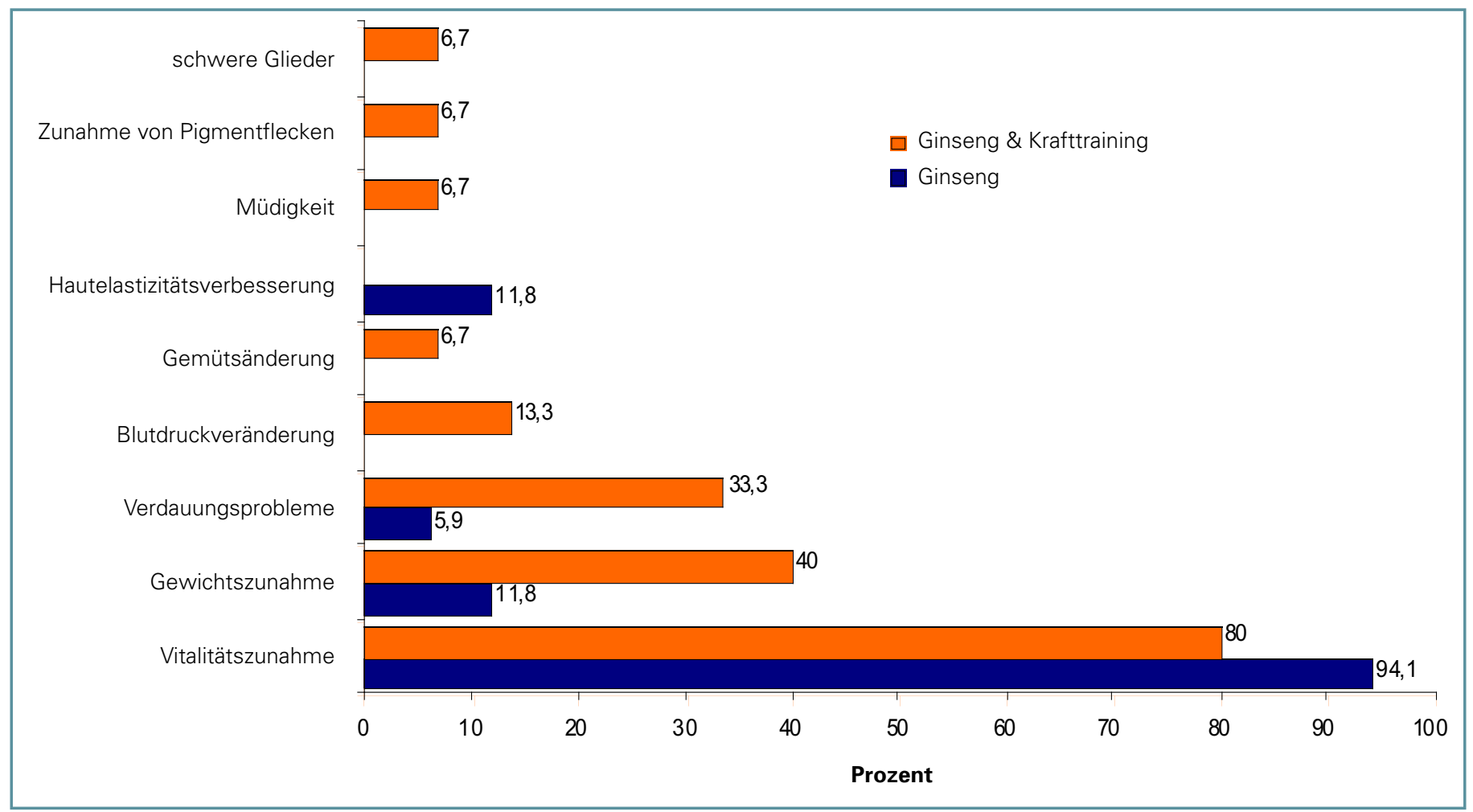

Abb. 1. Veränderung verschiedener Prüfparameter über die Treatmentdauer hinweg.

Untersuchung zwischen den Untersuchungsgruppen. In Abbildung 2a-f werden die deskriptiven Ergebnisse der Varianzanalyse mit dem Messwiederholungsfaktor Zeit, der unabhängigen Variablen 'Untersuchungsgruppe' sowie den Kovariaten 'Alter' und 'Geschlecht' für die Kraftparameter dargestellt. In der Kraft der Bauchmuskulatur profitierte insbesondere die Gruppe Ginseng \& Krafttraining mit einem Zuwachs von durchschnittlich $28 \%$, wobei die Probanden, die von einer Vitalitätsverbesserung sprachen, mit $33 \%$ vs. $19 \%$ eine noch deutlichere Zunahme verzeichnen konnten. Die reine Krafttrainingsgruppe zeigte eine Steigerung um 17\%. Der Haupteffekt Zeit $\left(F(1,64)=4.69, p=.034, \eta^{2}=.068\right)$ wurde hierbei signifikant ebenso wie die Wechselwirkung Gruppe $x$ Zeit $\left(\mathrm{F}(3,64)=7.84, \mathrm{p}<.001, \eta^{2}=.269\right)$. Die Kraft des oberen Rumpfes (überprüft am Rudergerät) konnte insgesamt um $5 \%$ gesteigert werden. Die Gruppe mit Vitalitätssteigerung erzielte durch die Bank höhere Ergebnisse. Wiederum ist dieses am deutlichsten für die Gruppe Ginseng \& Krafttraining mit 8\% bzw. $12 \%$. Die Krafttrainingsgruppe als auch die Ginsenggruppe mit Vitalitätsverbesserung steigerten ihre Leistung um $8 \%$. Neben den signifikanten Effekten für den Haupteffekt Zeit $(\mathrm{F}(1,64)=$ 15.68, $\left.\mathrm{p}<.001, \eta^{2}=.197\right)$ sowie die Wechselwirkung $(\mathrm{F}(3,64)=3.31, \mathrm{p}=.026$, $\eta^{2}=.134$ ) liegt ein signifikanter Effekt für die Kovariaten Alter $(\mathrm{F}(1,64)=8.77$, $\left.\mathrm{p}=.004, \quad \eta^{2}=.121\right)$ und Geschlecht $\left(\mathrm{F}(1,64)=6.83, \mathrm{p}=.011, \eta^{2}=.197\right)$ vor. An der Brustpresse gab es keine Verbesserungen für die Placebogruppe, jedoch deutliche Leistungssteigerungen für alle anderen Untersuchungsgruppen. Die Teilnehmer der Ginsenggruppe (mit Vitalitätsverbesserungen) konnten ihre Werte um $12 \%$ steigern, die Krafttrainingsgruppe um 24\% und die Gruppe Ginseng \& Krafttraining (mit Vitalitätsverbesserungen) um 27\%. Der Haupteffekt Zeit $(\mathrm{F}(1,64)=4.40$, $\mathrm{p}=.040, \eta^{2}=.064$ ) wurde hierbei signifikant ebenso wie die Wechselwirkung Gruppe x Zeit $(\mathrm{F}(3,64)=4.84, \mathrm{p}=.004$, $\left.\eta^{2}=.185\right)$. Die Kraft der Arme und der oberen Rückenmuskulatur, geprüft über den Latzug, wurde über alle Untersuchungsgruppen hinweg signifikant verbessert. Für die Wechselwirkung von Zeit und Gruppe $(F(3,64)=$
2.39, $\mathrm{p}=.077, \eta^{2}=.101$ ) lässt sich nur eine Tendenz beobachten, der Haupteffekt Zeit wird erwartungsgemäss signifikant $\left(F(1,64)=9.66, p=.003, \eta^{2}=\right.$ .131). Während sich die Placebo- und Ginsenggruppe gleichermassen um 8\% verbesserten, lag der Zuwachs für die Kraftgruppe bei $20 \%$ und bei der Gruppe Ginseng \& Krafttraining (mit Vitalitätsverbesserung) bei 15\%. Die Kraft der Kniebeuger (Beinpresse) konnte ebenfalls über die Treatmentdauer um $18 \%$ verstärkt werden. Während die Placebogruppe sich um $2 \%$ steigern konnte, sind diese Ergebnisse für die anderen Untersuchungsgruppen wesentlich deutlicher: Die Ginsenggruppe verbesserte sich um 9\%, die Krafttrainingsgruppe um 45\% und die Gruppe Ginseng \& Krafttraining in der Gruppe mit Vitalitätsverbesserung um 41\%. Eine statistisch bedeutsame Wechselwirkung liegt für Zeit $x$ Untersuchungsgruppe vor $\left(\mathrm{F}(3,64)=12.94, \quad \mathrm{p}<.001, \quad \eta^{2}=.378\right)$. Durchschnittlich um $15 \%$ wurde die Leistung bei den Kniestreckern verbessert, wobei dieses Ergebnis sich statistisch nicht bestätigen liess. Eine nur marginale Steigerung gab es für die 


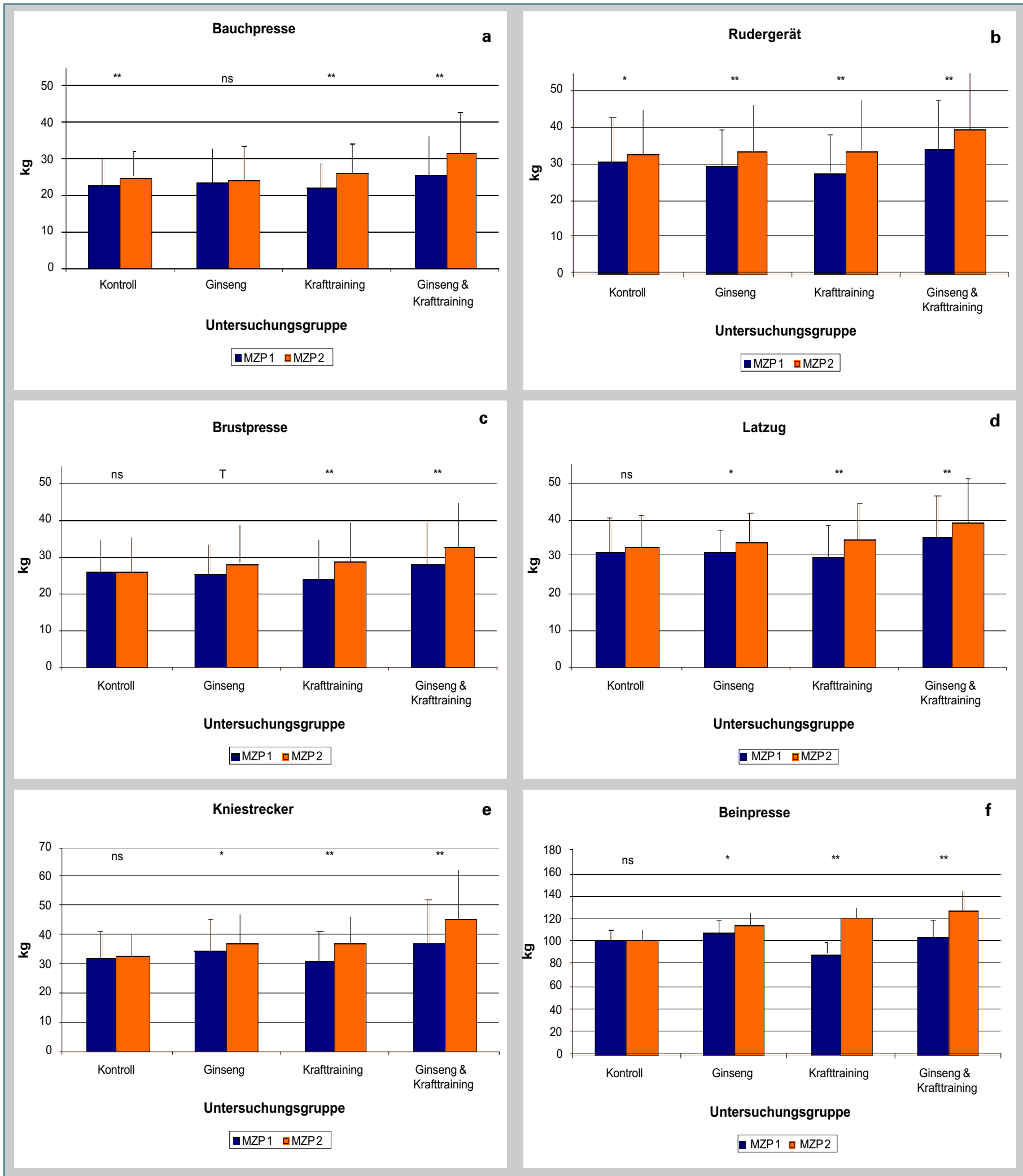

Abb. 2a-f. Deskriptive Ergebnisse bei den Kraftparametern in Abhängigkeit vom Messzeitpunkt (MZP) sowie der Untersuchungsgruppe. ns=nicht signifikant; $\mathrm{T}=\mathrm{p}<0.10 ;{ }^{*}=\mathrm{p}<0.05 ;{ }^{* *}=\mathrm{p}<0.01$

Placebogruppe. Die Ginsenggruppe konnte ihre Kraftwerte um $10 \%$ anheben, die Krafttrainingsgruppe um $21 \%$. Hinsichtlich der Treatmentgruppe
Ginseng \& Krafttraining ergab sich für die Gruppe ohne Vitalitätsverbesserung eine Leistungssteigerung um $24 \%$, für die Gruppe mit Vitalitätsver- besserung um $32 \%$. Die Wechselwirkung Zeit $x$ Untersuchungsgruppe kann als statistisch sehr bedeutsam eingestuft werden $\quad(F(3,64)=9.31$, 
$\left.\mathrm{p}<.001, \eta^{2}=.304\right)$. Das Alter der Teilnehmer erweist sich für das Rudergerät und den Latzug als signifikante Einflussvariable, Geschlechtseffekte finden sich für die Bauch- und Brustpresse, sowie das Rudergerät.

\section{Diskussion}

Die Ergebnisse der vorliegenden Studie stützen die deskriptiven Effekte von Rotem Ginseng auf klinische, Befindlichkeits- und Kraftparameter nach einem 12-wöchigen Krafttraining unter einer Gabe von 1200mg Rotem Ginseng mit einem Ginsengosidgehalt von $8 \%$ bei Erwachsenen im Alter von 52 bis 82 Jahren. Kritisch anzumerken bleibt, dass durch die Dropouts eine Ungleichverteilung hinsichtlich des Geschlechts erfolgte und keine weitere statistische Differenzierung möglich war.

Es ist wohlbekannt, dass insbesondere durch Krafttraining [3], aber auch durch die Gabe von Nahrungsergänzungsmitteln klinische Parameter [18] positiv beeinflusst werden können. Analog zu den Ergebnissen von MARASCo et al. [19] wurde eine leichte Gewichtszunahme in der reinen Ginsenggruppe beobachtet. Hinsichtlich der Pulsfrequenz als auch des Blutdrucks konnten sich alle Untersuchungsgruppen verbessern.

Positive Einschätzung einer Ginsenggabe durch Untersuchungsteilnehmer wurden von REVERs et al. [20], WIKLUND et al. [21] und MARAsco et al. [19] festgestellt. In der offenen Befragung der vorliegenden Studie - durchgeführt nach Ende des Treatments - berichteten nahezu alle Teilnehmer der Ginseng-Gruppe (80\%) sowie der Gruppe "Ginseng + Krafttraining” (94\%) von einer Vitalitätszunahme. Ähnliche Ergebnisse lassen sich für die Wirkungen eines Krafttrainings auf das Wohlbefinden feststellen [6]. Insbesondere jene Probanden, die von einer Vitalitätssteigerung berichteten (fast ausschliesslich die Probanden mit Ginsengeinnahme), zeigten die grössten $\mathrm{Zu}$ wächse in den Kraftleistungen.

Zahlreiche Studien belegen, dass Krafttraining auch bei Senioren in signifikanten Zunahmen in Muskelmasse als auch Muskelkraft resultiert und dieser Prozess über viele Monate hinweg ausgedehnt werden kann [22, 23]. Die Steigerung der Kraftwerte demonstriert die Fähigkeit zur Adaptation bei Erwachsenen höheren Alters [6], obwohl im Vergleich zu anderen Studien nur mittlere Intensitäten bewegt wurden und das Krafttraining nur einmal pro Woche durchgeführt wurde. Die Ergebnisse zeigen, dass Senioren in der Lage sind, ihre Kraft in den oberen und unteren Extremitäten als auch in der Rumpfmuskulatur zu steigern. Die deutlichsten Zuwächse zeigten die Versuchsteilnehmer für die Beinmuskulatur sowie die Bauchmuskulatur. MCNAUGHTON et al. [13] konnten in ihrer Untersuchung über einen Zeitraum von 6 Wochen Verbesserungen im M. pectoralis von $27 \%$ und im M. quadriceps von $18 \%$ feststellen. In der eigenen Untersuchung erzielten die Probanden der Ginseng \& Krafttrainingsgruppe an der Brustpresse (grosser Brustmuskel, Vorderer Teil des Deltamuskels, Langer Kopf des Triceps, Medialer Kopf des Triceps) ebenfalls eine Steigerung um 27\%. An der Beinpresse sowie bei den Kniestreckern konnten gar um 30 bzw. $40 \%$ bessere Werte erzielt werden. Insbesondere der Anstieg in den unteren Extremitäten durch ein entsprechendes Krafttraining ist durch die hohen Einbussen bei geringerer Beanspruchung im Alter zu erklären [24]. Verbesserungen in der Rumpfmuskulatur tragen insbesondere zu einer besseren Haltung sowie einer Abschwächung von Rückenschmerzen bei [3, 24]. Eine durchschnittliche Verbesserung um 28\% in der Ginseng- \& Krafttrainingsgruppe bzgl. der Bauchmuskulatur sowie die Berichte über eine bessere Haltung seitens der Versuchsteilnehmer bestätigen die Ergebnisse von DENNER [25], der mittels eines progressiv dynamischen Krafttrainings Abnahmen in Wirbelsäulenbeschwerden nachweisen konnte.

Betrachtet man den Alterungsprozess als Veränderung in den Kompetenzen (u.a. physisch, psychisch, kognitiv, sozial), so sollte in weiteren Studien neben einer Ausweitung von Treatmentdauer (mind. 6 Monate) und -umfang (2 bis 3 Trainingseinheiten mit einer Dauer von 60 bis 90 Minuten) sowie einer höheren Anzahl von Probanden auch eine kombinierte Form von körperlichem und kognitivem Training eingesetzt werden. Es sollte nicht nur die Kontrolle im Zuwachs von Kraftwerten vorgenommen werden: Für den älteren Menschen ist vor allem die Bewältigung des Alltags, also eine allgemeine Funktionstüchtigkeit des Systems Mensch von Interesse. Es hat sich gezeigt, dass schon mittlere Intensitäten zu Leistungszuwächsen führten. Unter praktischen Gesichtspunkten gilt es nun zu prüfen, ob auch ein Training mit freien Gewichten, Therabändern u.ä. zu gleichen Ergebnissen führt. Denn nicht allen Senioren ist es möglich, ein Fitnessstudio o.ä. aufzusuchen. Bei Berücksichtigung kognitiver Leistungsfähigkeit, wie es u.a. in Studien von SorEnson und SonNe [26] vorgenommen wurde, sollte eine Ginsenggabe von $1200 \mathrm{mg}$ pro Tag (Ginsenosidgehalt mindestens $8 \%$ ) beibehalten werden.

\section{Danksagung}

Die für die vorliegende Studie benötigte Prüfmedikation (Verum und Placebo) wurde freundlicherweise zur Verfügung gestellt von der Firma Gintec International GmbH, DE-61462 Königstein.

\section{Literatur}

1. Statistisches Bundesamt: Bevölkerungsentwicklung Deutschlands bis zum Jahr 2050. Ergebnisse der 9. koordinierten Bevölkerungsvorausberechnung des Bundes und der Länder zur Bevölkerungsentwicklung bis 2050. Wiesbaden, statistik-bund.de 2000.

2. Nikolaus T: Einfluss körperlicher Aktivität auf funktionelle Fähigkeiten. Zeitschrift für Gerontologie und Geriatrie 2001;34:44-47.

3. Schott N: Bewegung und Sport im Alter. In: Zentgraf K (Hrsg.), Schlaganfall. Hofmann, Schorndorf 2003, S. 91-106.

4. Bös K, Abel T, Woll A, Niemann S, Tittlbach S, Schott N: Der Fragebogen zur Erfassung des motorischen Funktionsstatus (FFB-MOT). Diagnostica, 2002;48(2),101-111.

5. Roubenoff R: Sarcopenia and its implications for the elderly. European Journal of Clinical Nutrition, 2000;54(Suppl),40-47.

6. Cavani V, Mier CM, Musto AA, Tummers N Effects of a 6-Week Resistance-Training Programm on Functional Fitness of Older Adults Journal of Aging and Physical Activity 2002 10,443-452.

7. Allmer $\mathrm{H}$ : Bewegungs- und Sportaktivitäten Älterer aus psychologischer Sicht - Wissen wir schon alles? In: Daugs $R$, Emrich $E$, Igel C, Kindermann W (Hrsg.): Áktivität und Altern. Hofmann, Schorndorf 2001, S. 201-210.

8. Radad K, Gille G, Liu L, Rausch W-D: Use of 
Ginseng in Medicine With Emphasis on Neurodegenerative Disorders. J Pharmacol Sci 2006; 100,175-186.

9. European Pharmacopoeia Ph. Eur. 5.0 (2005) www.pheur.org

10. Bucci LR: Selected herbals and human exercise performance. American Journal of Clinical Nutrition, 2000;72(2), 624S-636s.

11. Engels HJ, Wirth JC: No ergogenic effects of ginseng (Panax ginseng C.A. Meyer) during graded maximal aerobic exercise. J. Am. Diet. Assoc. 1997:97,1110-1115.

12. Pieralisi G, Ripari P, Vecchiet L: Effects of a standardized ginseng, extract combined with dimethylaminoethanol bitartrate, vitamins, minerals, and trace elements on physical performance during exercise. Clin Ther 1999,13,373-382.

13. McNaughton L, Egan G, Caelli G: A comparison of Chinese and Russian ginseng as ergogenic aids to improve various facets of physical fitness. Int Clin Nutr Rev 1989;90,32-35.

14. Kang HY, Kim SH, Lee WJ, Byrne HK: Effects of Ginseng ingestion on growth hormone, testosterone, cortisol, and insulin-like growth factor 1 responses to acute resistance exercise. Journal of Strength and Conditioning Research 2002;16 (2),179-183.

15. Bahrke MS, Morgan WP: Evaluation of the
Ergogenic Properties of Ginseng. Sports Medicine, 2000;29(2),113-133.

16. Salem GJ, Wang M-Y, Sigward S: Measuring Lower Extremity Strength in Older Adults: The Stability of Isokinetic Versus 1RM Measures. Journal of Aging and Physical Activity 2002;10,489-503.

17. Borg GAV: Psychophysical bases of perceived exertion. Medicine Science \& Sports Exercise 1982;14,377-381.

18. Engels H-J, Fahlman MM, Wirth, JC: Effects of Ginseng on Secretory IgA, Performance, and Recovery from Interval Exercise. Med. Sci. Sports Exerc., 2003,35(4),690-696.

19. Marasco C, Vargas R, Villagomez S, Infante B: Double-blind study of a multivitamin complex supplemented with ginseng extract. Drugs Exp Clin Res 1996;22,323-329.

20. Revers W, Simon WCM, Popp F et al. : Psychological effects of a geriatric preparation in the aged. Z Prakt Klin Geriat 1976;9,418-30.

21. Wiklund I, Karlberg J, Lund B: A double-blind comparison of the effect on quality of life of a combination of vital substances including standardized ginseng G115 and placebo. Curr Ther Res 1994;55,32-42.

22. Häkkinen $K$, Kallinen $M$, Izquierdo $M$, Jokelainen $\mathrm{K}$, Lassila $\mathrm{H}$, Mälkiä $\mathrm{E}$, et al.: Changes in agonist-antagonist EMG, muscle CSA, and
Originalarbeit | Original Article

force during strength training in middle-aged and older people. Journal of Applied Physiology 1998;84,1341-1349

23. Schott N, Knobl O: The effects of free weights training for older adults. Journal of Aging and Physical Activity 2004;12,413-414

24. Spirduso W, Francis KL, MacRae PG: Physical dimensions of aging (2nd. ed.). Human Kinetics, Champaign, IL 2005.

25. Denner A: Die Trainierbarkeit der Rumpfmus kulatur älterer Menschen mittels progressiv dynamischem Krafttraining und Funktionsgymnastik. Physiotherapie 1995;86,409-410.

26. Sorensen $H$, Sonne J: A double-masked study of the effects of ginseng on cognitive functions. Curr Ther Res 1996;57,959-68.

\section{Korrespondenzadresse:}

Dr. phil. Nadja Schott,

Institut für Sportwissenschaft, Justus-Liebig Universität Giessen, Kugelberg 62, DE-35394 Giessen

Nadja.Schott@sport.uni-giessen 